\title{
Effet du mode de distribution de la ration et de la forme de présentation des aliments sur l'aminoacidémie plasmatique chez le poney
}

\author{
L Cabrera $^{1 *}$, C Durier ${ }^{2}$, JL Tisserand ${ }^{1 \star \star}$ \\ 1 Unité associée de recherches zootechniques Enesad-Inra, BP 1607, 21036 Dijon cedex; \\ 2 Laboratoire de biométrie, Centre de recherche Inra de Versailles, \\ route de Saint-Cyr, 78026 Versailles cedex, France
}

(Reçu le 2 novembre 1993 ; accepté le 1er juin 1994)

\begin{abstract}
Résumé - Nous avons étudié l'effet du mode et du rythme de distribution d'aliments agglomérés ou non sur l'aminoacidémie chez le poney, les rations sont composées de paille mélassée $(68 \%)$ et de concentré ( $25 \%$ maïs grain $+7 \%$ tourteau de soja) distribuées au niveau de l'entretien. Dans un premier essai, nous avons utilisé huit poneys mâles adultes (d'un poids moyen de $202 \mathrm{~kg}$ ), selon un plan en cross-over $2 \times 2$. Les rations sont ainsi distribuées : $R 1$ : paille mélassée hachée et concentré aggloméré distribués en même temps, en deux repas, à $8 \mathrm{~h}$ et à $17 \mathrm{~h} ; \mathrm{R} 2$ : concentré aggloméré distribué 2 heures après le repas de paille mélassée hachée, en deux repas, soit $8 \mathrm{~h}$ et $16 \mathrm{~h}$ pour la paille et $10 \mathrm{~h}$ et $18 \mathrm{~h}$ pour le concentré. Dans le deuxième essai, neuf poneys adultes (d'un poids moyen de $198 \mathrm{~kg}$ ) sont utilisés selon un dispositif en carré latin ; trois régimes ont été comparés : R3 : paille mélassée plus concentré sous forme d'un seul aggloméré distribué à $8 \mathrm{~h}$ et $17 \mathrm{~h} ; \mathrm{R} 4$ : paille mélassée agglomérée plus concentré aggloméré distribué en même temps ( $8 \mathrm{~h}$ et $17 \mathrm{~h}$ ); R5 : paille mélassée agglomérée ( $8 \mathrm{~h}$ et $16 \mathrm{~h}$ ) plus concentré aggloméré offert 2 heures après le repas de paille mélassée (10 h et $18 \mathrm{~h}$ ). Des prises de sang ont été effectuées au niveau de la veine jugulaire à $8 \mathrm{~h}(T), 10 \mathrm{~h}(T+2)$, $11 \mathrm{~h}(T+3), 13 \mathrm{~h}(\mathrm{~T}+5), 14 \mathrm{~h}(\mathrm{~T}+6), 16 \mathrm{~h}(\mathrm{~T}+8), 17 \mathrm{~h}(\mathrm{~T}+9)$ afin de doser le taux plasmatique en acides aminés totaux (TPAA). Les résultats montrent que R1 est légèrement supérieur à $R 2$, la moyenne journalière TPAA s'établissant respectivement à 2687 et $2484 \mu \mathrm{mol} / \mathrm{L}$, avec des pics à T+2 (3639 $\mathrm{mmol} / \mathrm{L}$ de sang) pour $\mathrm{R} 1$ et à $T+6(2712 \mu \mathrm{mol} / \mathrm{L})$ pour $\mathrm{R} 2$. En revanche, pour l'essai 2 , le TPAA de R5 est supérieur à ceux de R4 et R3 (respectivement 2745,2608 et $2525 \mu \mathrm{mol} / \mathrm{L}$ ) avec des pics de TPAA à $T+6(3087 \mu \mathrm{mol} / \mathrm{L}) ; T+2(3443 \mu \mathrm{mol} / \mathrm{L})$ et à $T+2(2914 \mu \mathrm{mol} / \mathrm{L})$. L'aminoacidémie reste élevée plus longtemps dans le cas de régime dissocié. L'effet favorable de la distribution du concentré après le repas de paille sur la digestion de l'azote semble se confirmer, mais il pourrait être atténué lorsque le concentré est aggloméré.
\end{abstract}

poney / nutrition / acide aminé plasmatique / mode de distribution

* Boursière du CNPq (Brésil)

** Correspondance et tirés à part 
Summary - Influence of the physical form of feeds and of the time of feeding concentrate on plasma concentrations of amino acids in ponies fed straw and concentrate diets. A diet consisting of molassed straw (68\%) and concentrate (25\% maize and $7 \%$ soya-bean meal) was fed to achieve INRA recommandations for maintenance. In a first experiment, E1, a $2 \times 2$ crossover design was used with eight adult male ponies (average weight $202 \mathrm{~kg}$ ) to compare two different diets: $R 1$ : pelleted concentrate and molassed straw fed simultaneously (at 800 and 1600 hours); R2: pelleted concentrate fed two hours after the molassed straw (at 1000 and 1800 hours). In a second experiment, E2, a $3 \times 3$ latin square design was used with nine adult male ponies (average weight $198 \mathrm{~kg}$ ) to compare three different diets: R3: combined concentrate and molassed straw pellets (fed at 8.00 and 1600 hours); R4: pelleted concentrate and pelleted molassed straw fed simultaneously (at 8.00 and 1600 hours); R5: pelleted concentrate fed two hours after the pelleted straw (at 10.00 and $18.00 \mathrm{~h}$ ). Samples of jugular blood were taken at $800(T), 1000(T+2), 1100(T+3), 1300(T+5), 1400(T+6), 1600(T+8), 1700(T+9)$ hours, for determination of plasma free amino acids (PFAA). $R 1$ gave higher concentrations of total PFAA than R2. Daily average concentrations were 2687 with $R 1$ and $2484 \mu$ mol/L with $R 2$. Peak values were reached at $T+2$ with $R 1(3639 \mu \mathrm{mol} / \mathrm{L})$ and at $T+6$ with $R 2(2712 \mu \mathrm{mol} / \mathrm{L})$. In E2, daily average concentrations were higher with $R 5$ (2 $745 \mu \mathrm{mol} / \mathrm{L})$ than with $R 4(3443 \mu \mathrm{mol} / \mathrm{L})$ and $R 3(2608 \mu \mathrm{mo} / \mathrm{L})$. Peak values were reached at $T+6(3087 \mu \mathrm{mol} / \mathrm{L}), T+2(3443 \mu \mathrm{mol} / \mathrm{L})$ and $T+2(2914 \mu \mathrm{mol} / \mathrm{L})$, respectively. The positive effect on nitrogen digestion of feeding the concentrate after the straw seems to be affirmed, but could be less pronounced if the concentrate is pelleted

ponies / nutrition / plasma amino acid / feed

\section{INTRODUCTION}

Les dépenses azotées des équidés sont couvertes par les acides aminés (AA) absorbés lors de la digestion des aliments, essentiellement dans l'intestin grêle (Glade, 1983; Gibbs et al, 1988 ; Potter et al, 1992).

L'apport azoté alimentaire dépend tout autant de la quantité que de la qualité des protéines. Plus les proportions en AA indispensables d'une protéine alimentaire sont proches des proportions des protéines constitutives des différents tissus, plus la qualité des protéines est élevée (Frape, 1986). Le taux plasmatique en AA (TPAA) peut être alors considéré comme un témoin satisfaisant de la qualité azotée de la ration.

Différents auteurs (Johnson et Hart, 1974 ; Russel et al, 1986 ; Gibbs et al, 1988) montrent que, chez les équidés, l'aminoacidémie plasmatique est un indicateur satisfaisant de la quantité et de la qualité de l'azote absorbé au niveau de l'intestin grêle. Le TPAA est d'autant plus élevé que la source de protéine est de bonne qualité (Reitnour et al, 1970), ou que la ration est distribuée en plusieurs repas (Johnson et Hart, 1974).

Une étude réalisée précédemment (Cabrera et Tisserand, 1995) montre, plus particulièrement, que la distribution d'un concentré riche en azote (maïs grain plus tourteau de soja non agglomérés), 2 heures après le repas de fourrage grossier (paille), augmente l'aminoacidémie plasmatique. La distribution du même régime totalement aggloméré entraîne un taux moyen journalier d'acides aminés essentiels intermédiaire entre la distribution associée ou dissociée des aliments du régime.

L'objet de ce travail est d'étudier l'effet de l'agglomération du concentré distribué en complément de paille mélassée hachée ou agglomérée offerts séparément ou ensemble sur l'aminoacidémie chez le poney, pour préciser les résultats précédemment obtenus.

\section{MATÉRIEL ET MÉTHODES}

Deux essais indépendants, avec deux rations de taux azotés différents, sont réalisés sur des 
poneys adultes mâles entiers de race Shetland. Leurs besoins nutritionnels sont calculés par référence aux recommandations francaises pour les chevaux (Inra, 1984 ; Martin-Rosset, 1994), en énergie $\left(0,038 \mathrm{UFC} / \mathrm{kg} \mathrm{P}^{0,75}\right)$ et en azote $(2,8 \mathrm{~g}$ MADC $/ \mathrm{kg} \mathrm{P}^{0,75}$ ). Les animaux sont adaptés aux régimes expérimentaux pendant une période de 14 jours avant une journée de mesures. Durant cette période, ils sont maintenus en box puis ils sont mis à l'attache dans des stalles au cours de la journée de mesures, afin d'éviter toute consommation de paille de litière. De l'eau de boisson et des pierres à sel sont mises à leur disposition.

Les régimes sont composées de $68 \%$ de paille de blé hachée mélassée (10\% de mélasse de betterave) agglomérée ou non et de $32 \%$ d'un aliment concentré aggloméré constitué de $78 \%$ maïs grain et $22 \%$ tourteau de soja 44 . Les rations sont calculées pour couvrir les besoins énergétique d'entretien, ce qui entraîne un niveau d'apport azoté supérieur de $30 \%$ à celui de l'entretien, compte tenu de la nature des aliments utilisées.

La composition chimique des aliments est donnée dans le tableau I.

\section{Essai I}

Cet essai comporte deux périodes expérimentales afin de tester deux rythmes différents de distribution du même régime :

Tableau I. Composition chimique des aliments.
- R1 : fourrage haché et concentré aggloméré distribués ensemble, en deux repas ( $8 \mathrm{~h}$ et $17 \mathrm{~h}$ );

- R2 : concentré aggloméré distribué 2 heures après la paille hachée distribuée à $8 \mathrm{~h}$ et à $16 \mathrm{~h}$, puis retirée à $10 \mathrm{~h}$ et à $18 \mathrm{~h}$ avant la distribution du concentré.

Les mesures sont effectuées sur huit poneys (pesant en moyenne $203 \pm 16 \mathrm{~kg}$ en début d'expérience) répartis aléatoirement en deux groupes de quatre selon un plan en cross-over $2 \times 2$, ce qui permet une bonne interprétation des résultats.

Les rations journalières sont composées de $2535 \mathrm{~g}$ de paille mélassée hachée et de $1194 \mathrm{~g}$ de concentré aggloméré.

\section{Essai 2}

Dans le deuxième essai nous expérimentons un aggloméré complet (R3) et deux agglomérés (fourrage et concentré) distribués en même temps (R4) ou selon le rythmes d'une étude précédente (Cabrera et Tisserand, 1995).

Pour chacune de ces trois périodes, neuf poneys pesant en moyenne $198 \pm 17 \mathrm{~kg}$ sont répartis de façon aléatoire par groupe de trois selon un dispositif en carré latin $3 \times 3$ en mesures répétées.

Les rations journalières sont distribuées à raison de $2498 \mathrm{~g}$ de paille mélassée agglomérée et

\section{Constituants}

$\begin{array}{clcc}\text { MS } & \text { Cendres } & C B & \text { MAT } \\ (\% \text { brut }) & (\% M S) & (\% M S) & (\% M S)\end{array}$

\section{Essai 1}

Paille mélassée hachée

90,2

88,3

7,7

39,2

5,4

Concentré aggloméré

(78\% maïs $+22 \%$ tourteau soja 44$)$

\section{Essai 2}

Paille mélassée agglomérée

Concenté aggloméré

( $78 \%$ maïs $+22 \%$ tourteau soja 44 )

Aggloméré complet

( $68 \%$ paille mélassée $+25 \%$ maïs $+7 \%$ tourteau soja 44 )

$\begin{array}{rrrr}90,4 & 7,3 & 37,5 & 5,9 \\ 88,1 & 2,8 & 3,7 & 21,4 \\ & & & \\ 88,5 & 6,2 & 23,9 & 11,0\end{array}$


$1174 \mathrm{~g}$ de concentré aggloméré, en deux repas par jour : R3 et R4: $8 \mathrm{~h}$ et $17 \mathrm{~h} ; \mathrm{R} 5: 8 \mathrm{~h}$ et $16 \mathrm{~h}$ pour la paille agglomérée ; $10 \mathrm{~h}$ et $18 \mathrm{~h}$ pour le concentré.

\section{Les mesures et analyses}

Le paramètre mesuré dans ces essais est l'aminoacidémie plasmatique. Les conditions de prélèvement et de traitement du sang prélevé à la veine jugulaire d'une part et celles du dosage des acides aminés d'autre part ont été décrites précédemment par Cabrera et Tisserand (1995).

Dans le présent travail sept prélèvements sont réalisés afin d'augmenter la précision de la mesure : le premier à jeun à $8 \mathrm{~h}$, juste avant le repas puis à $10,11,13,14,16$ et $17 \mathrm{~h}$ (soit $T$, $T+2, T+3, T+5, T+6, T+8$ et $T+9$ ).

\section{Traitement statistique des résultats}

Les données sont traitées statistiquement par une procédure d'analyse de variance (au moyen du logiciel Sas, 1988). Les variables expliquées sont les teneurs en acides aminés des sept prélèvements de la journée, traités séparément, et leur valeur moyenne sur la journée.

Le modèle usuel pour ce dispositif expérimental (Winer, 1971) s'écrit :

$$
Y_{i j k l}=\mu+a_{i}+t l_{(i)}+b_{j}+g_{k}+b_{g j k}+e_{i j k l}
$$

avec $Y$ : variable expliquée; $i$ : groupe ; $j$ : régime; $k$ : période $; l_{(i)}$ : poney $/$ du groupe $i ; a_{i}$ : effet du groupe $i ; t_{(i)}$ : effet du poney /emboîté dans l'effet du groupe $i$, terme considéré comme erreur pour l'effet groupe $i ; b_{j}$ : effet du régime $j ; g_{k}$ : effet de la période $k ; b_{g j k}$ : interaction régime $\mathrm{x}$ période; $e_{i j k l}:$ erreur pour les effets régime, période, interaction.

Grâce au tableau d'analyse de variance associé à ce modèle, il est possible d'effectuer des tests de Fisher sur l'existence de différences entre les effets des régimes, des périodes ou sur l'existence d'une interaction régime $\mathrm{x}$ période à un niveau fixé de $95 \%$.

\section{RÉSULTATS}

En tenant compte des refus de paille mélassée enregistrés, les niveaux énergétique et azoté (apport total/besoin d'entretien) des régimes réellement ingérés sont présentés dans le tableau II. Ils sont en accord avec notre objectif de rationnement si nous considérons a priori qu'il n'y a pas d'interactions digestives entre les différents éléments du régime compte tenu de leur mode de présentation et des rythmes de distribution.

Tableau II. Niveau énergétique et azoté fournis par les rations consommées.

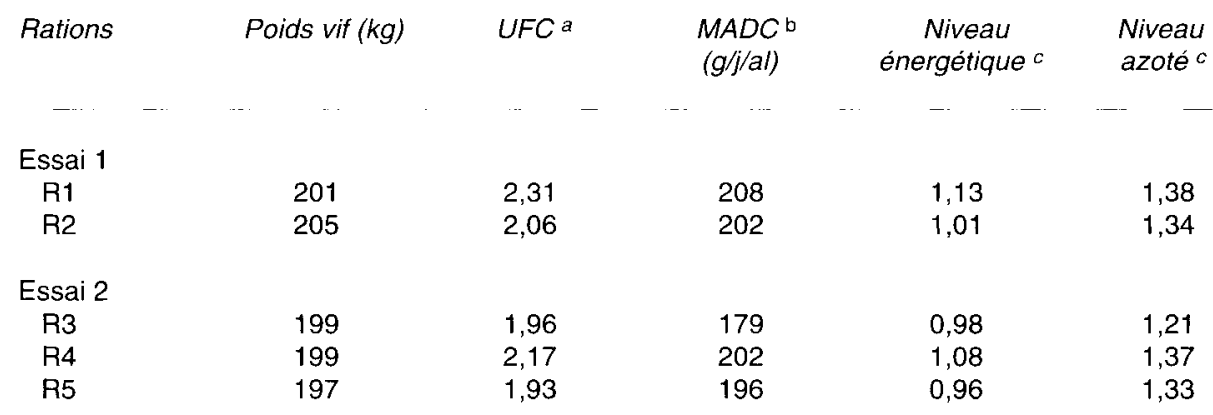

a Unité fourragère cheval ; $b$ matière azotée digestive cheval (systèmes Inra, 1984); ${ }^{c}$ niveau : apport total / besoins entretien. 


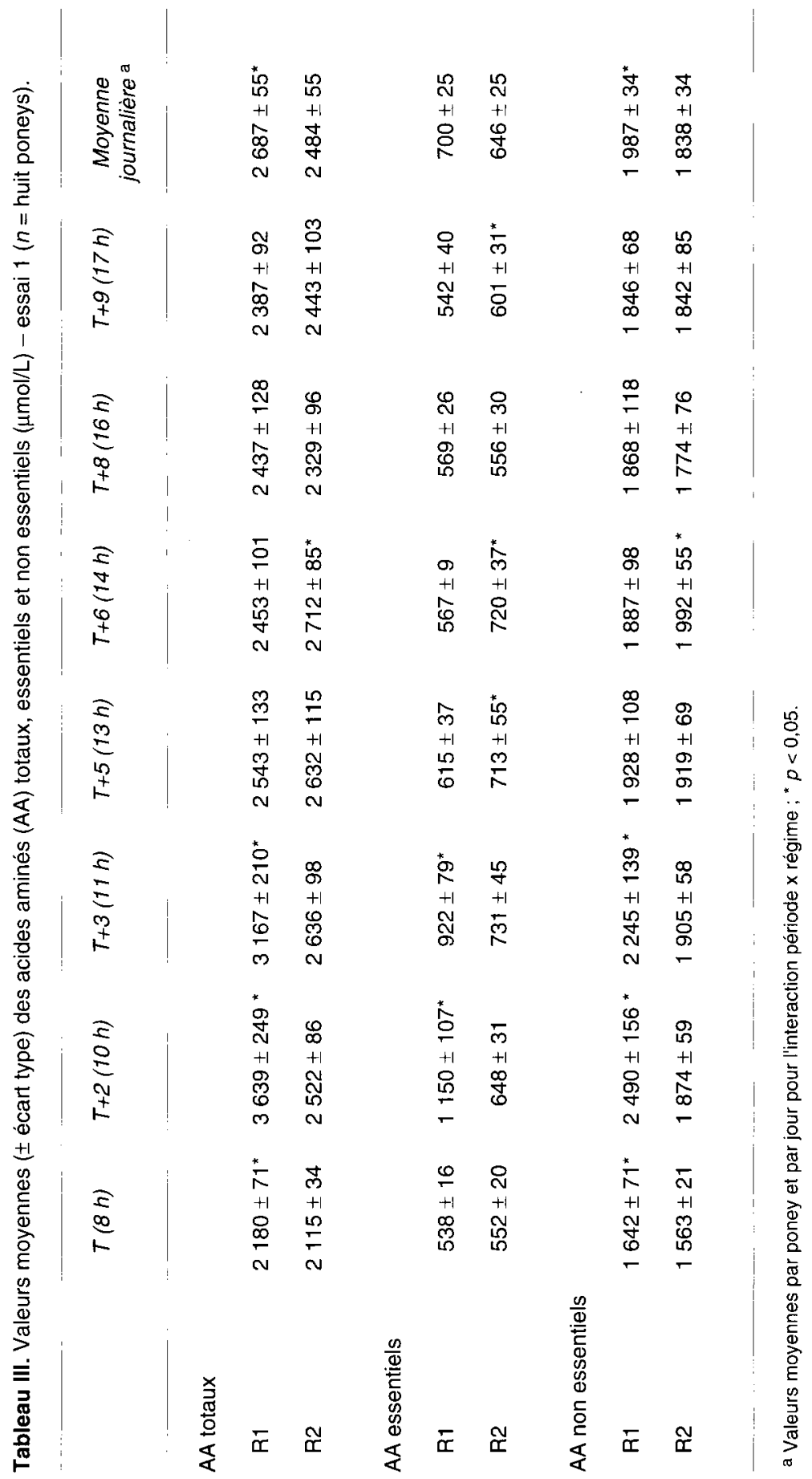




\section{Essai 1 (tableau III)}

L'interaction période $x$ régime n'a pas été étudiée dans cet essai parce qu'elle est confondue avec l'effet groupe.

Les taux plasmatiques moyens journaliers en $A A$ totaux (AAT) et AA non essentiels (AANE) des poneys R1 sont significativement supérieurs à ceux mesurés chez les poneys $\mathrm{R} 2$ de $+7,6 \%$ et de $+7,5 \%$ respectivement. La différence est essentiellement due à deux pics importants d'aminoacidémie observés à $T+2$ et à $T+3$.

En revanche les concentrations en AAT et AANE des poneys $R 2$ sont plus élevées au cours des 6 heures suivant la distribution du concentré $(T+5, T+9)$ (tableau III). Mais, à jeun, le taux d'AAE est supérieur bien que non significativement pour R2 par rapport à $\mathrm{R} 1$.

\section{Essai 2 (tableau IV)}

Le régime $\mathrm{R} 5$ (distribution dissociée) entraîne une aminoacidémie totale moyenne journalière des poneys significativement supérieure $(+8 \%)$ à celle des poneys R3 (distribution d'un seul aggloméré) mais non significativement supérieure $(+5 \%)$ à celle des animaux R4 (les deux agglomérés distribués en même temps). La différence observée est aussi bien liée aux AA essentiels qu'aux AA non essentiels. Elle est particulièrement marquée à partir de $T+5$ ( 3 heures après la distribution du concentré).

L'aminoacidémie totale à $T+2$, pour R3 et R4, est logiquement supérieure à celle de de R5 car, dans ce dernier cas, les animaux n'ont pas encore reçu le concentré. À T+3, l'aminoacidémie générée par les trois régimes est comparable ( 2814,2931 et 2 $894 \mu \mathrm{mol} / \mathrm{L}$ respectivement pour R3, R4 et pour R5).

Toutefois l'importance du pic à $T+2$ de R4 par rapport à $\mathrm{R} 3$ indique que, lorsqu'on donne en même temps les deux aliments agglomérés, les poneys consomment préférentiellement l'aggloméré concentré.

L'analyse de variance ne révèle pas ou peu d'interactions entre les périodes et les régimes $(p<0,04 ; p>0,13$ et $p>0,34)$ pour les valeurs d'aminoacidémie moyenne journalière.

Aux points de cinétique $T+2, T+3$ et $T+8$ l'interaction période $x$ régime est significative à $95 \%$ par rapport à l'erreur résiduelle. Les régimes ont donc des effets différents selon les périodes. Avec ce dispositif, l'interaction est partiellement confondue avec l'effet groupe. Le classement des régimes aux différentes périodes est très délicat et impossible avec les logiciels couramment disponibles. Cependant, l'effet significatif du régime $\mathrm{R} 5$ semble probable. Un plan d'expérience légèrement différent, par exemple fondé sur deux carrés latins orthogonaux à la place d'un seul carré latin, aurait permis un classement des moyennes des régimes aux trois périodes (John, 1971).

En revanche, pour $T+5, T+6$ et $T+9$, l'interaction période $x$ régime n'est plus significative ; il est donc possible de comparer les régimes indépendamment des périodes. L'aminoacidémie générée par R5 (régime dissocié) apparaît alors significativement supérieure à celle de R3 et R4.

\section{DISCUSSION}

Les rations étudiées sont distribuées au niveau de l'entretien pour l'énergie et de 1,3 des besoins de l'entretien pour l'azote, si on considère que les effets éventuels des traitements mécaniques et de la forme de présentation des fourrages et des aliments concentrés sur la digestibilité sont limités, voire contradictoires, dans l'état actuel des connaissances (Martin-Rosset et al, 1984). L'aminoacidémie moyenne journalière des animaux recevant un concentré sous forme 


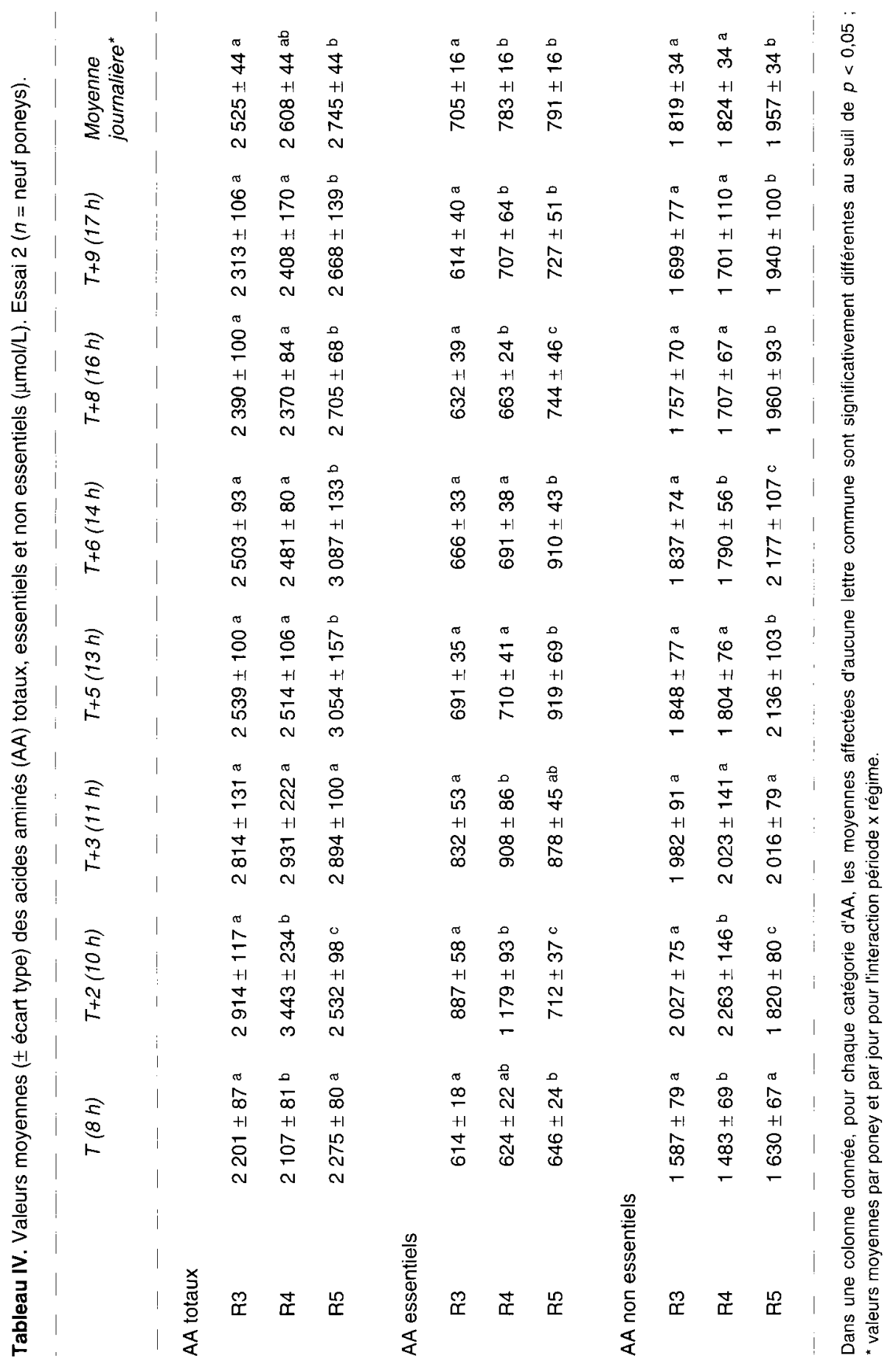


agglomérée 2 heures après le repas de paille mélassée hachée, n'a pas été supérieure à celle des animaux recevant les mêmes aliments simultanément, contrairement à ce que nous avons précédemment mesuré avec un concentré brut et non aggloméré (Cabrera et Tisserand, 1995). La différence observée est sans doute due à une interaction broyage et agglomération ou à un effet favorable de la finesse de broyage.

Dans l'essai 2, l'aminoacidémie moyenne journalière de $\mathrm{R} 5$ fournit des résultats cohérents avec nos précédents travaux (Cabrera et Tisserand, 1995).

Dans le présent travail l'aminoacidémie persiste plus longtemps à un niveau plus élevé $(+10 \%)$ dans le cas du régime dissocié $\mathrm{R} 2$ comparativement au régime associé R1.

La persistance de l'aminoacidémie peut paraître plus significative d'un point de vue physiologique qu'une moyenne journalière souvent déterminée, en grande partie, par un pic précoce d'aminoacidémie comme nous le remarquons à $T+2$ et à $T+3$ pour R1 (essai 1). Il est possible d'appliquer le même raisonnement pour l'essai 2 où la paille est distribuée sous forme agglomérée, ce qui entraîne des pics pour $\mathrm{R} 3$ et surtout pour R4 se situant à $T+2$.

Il semble globalement que, quelles que soient les caractéristiques physiques des aliments étudiés (dans le présent travail : paille hachée ou agglomérée et concentré aggloméré distribués séparément ou simultanément et dans le précédent travail de Cabrera et Tisserand, 1995 : paille hachée et concentré non aggloméré), la distribution du concentré après le repas de paille permet une meilleure disponibilité des matières azotées de la ration, sans doute grâce à un séjour plus long de la fraction concentré dans l'estomac et à un transit moins rapide dans l'intestin grêle. Mais la paille hâchée peut accélérer le transit, indépendamment de la quantité distribuée dans la ration (Gul- denhaupt, 1979). Cet effet paraît, dans les conditions de notre étude, fortement atténué par le broyage du concentré azoté, qui pourrait favoriser une digestion plus rapide dans l'intestin grêle.

\section{CONCLUSION}

L'effet favorable de la distribution différée du concentré après le repas de paille sur la digestion de l'azote semble se confirmer, mais il est atténué lorsque le concentré broyé est aggloméré.

La distribution différée des aliments agglomérés aurait également comme avantage d'allonger la période d'absorption de l'azote de la fraction "concentré" et de réduire la variabilité de l'aminoacidémie au cours de la journée.

Les mesures du temps de transit digestif des aliments que nous avons entreprises devraient permettre de mieux expliquer les différences observées.

\section{RÉFÉRENCES}

Cabrera L, Tisserand JL (1995) Effet du rythme de distribution et de la forme de présentation d'un régime paille-concentré (à deux niveaux d'apport azoté) sur l'aminoacidémie chez le poney. Ann Zootech 44, 105114

Frape D (1986) Equine nutrition and feeding. Longman Scientific and Technical, New York, $373 p$

Gibbs PG, Potter GD, Shelling GJ, Kreider ZL, Boyd CL (1988) Digestion of hay protein in different segments of the equine digestive tract. J Anim Sci66, 400-406

Glade MJ (1983) Nitrogen partioning along the equine digestive tract. J Anim Sci 57, 943-953

Güldenhaupt V (1979) Verträglichkeit und verdaulichkeit eines alleinfutters für pferde in kombination mit stroh. Thèse de doctorat, École vétérinaire, Hannovre, $100 p$

Inra (1984) Le cheval (R Jarrige, W Martin-Rosset, eds), Inra Publications, Paris, $689 \mathrm{p}$

John PWM (1971) Statistical design and analysis of experiments. MacMillan, New York, États-Unis, $365 \mathrm{p}$

Johnson RJ, Hart JW (1974) Influence of feeding and fasting on plasma free amino acids in the equine. JAnim Sci 38, 790-794 
Martin-Rosset W, Andrien J, Vermorel M, Dulphy JP (1984) Valeur nutritive des aliments pour le cheval. In : Le cheval (R Jarrige, W Martin-Rosset, eds), Inra Publications, Paris, 207-238

Martin-Rosset $\mathrm{W}$, Vermorel M, Doreau M, Tisserand $\mathrm{JL}$, Andrien J (1994) The French horse feed evaluation systems and recommanded allowances for energy and protein. Livest Prod Sci 40, 37-56

Potter GD, Gibbs PG, Haley RG, Klendsho JC (1992) Digestion of protein in the small and large intestines of equines fed mixed diets. 1st Europäische Konfe- renz über die ernährung des pferdes. Pferdeheilkunde, 140-143

Reitnour CM, Baker JP, Mitchell Jr GE, Little CO, Kratzer DD (1970) Amino acids in equine cecal content, cecal bacteria and serum. J Nutr 100, 349-354

Russel MA, Rodiek AV, Lawrence LM (1986) Effect of meal schedules and fasting on selected plasma free amino acids in horses. J Anim Sci63, 1428-1431

SAS (1988) SAS/Stat user's guide. SAS Institute Inc, Cary, NC, États-Unis, $1028 \mathrm{p}$

Winer BJ (1971) Statistical principals in experimental design. McGraw-Hill, New York, États-Unis, 907 p 